\title{
RESEARCH
}

Open Access

\section{Mimicry and well known genetic friends: molecular diagnosis in an Iranian cohort of suspected Bartter syndrome and proposition of an algorithm for clinical differential diagnosis}

Maryam Najafi ${ }^{1,2}$, Dor Mohammad Kordi-Tamandani ${ }^{2 *}$, Farkhondeh Behjati ${ }^{3}$, Simin Sadeghi-Bojd ${ }^{4}$, Zeineb Bakey ${ }^{1,8}$, Ehsan Ghayoor Karimiani ${ }^{5,6}$, Isabel Schüle ${ }^{8}$, Anoush Azarfar ${ }^{7}$ and Miriam Schmidts ${ }^{1,8,9^{*}}$ (D

\begin{abstract}
Background: Bartter Syndrome is a rare, genetically heterogeneous, mainly autosomal recessively inherited condition characterized by hypochloremic hypokalemic metabolic alkalosis. Mutations in several genes encoding for ion channels localizing to the renal tubules including SLC12A1, KCNJ1, BSND, CLCNKA, CLCNKB, MAGED2 and CASR have been identified as underlying molecular cause. No genetically defined cases have been described in the Iranian population to date. Like for other rare genetic disorders, implementation of Next Generation Sequencing (NGS) technologies has greatly facilitated genetic diagnostics and counseling over the last years. In this study, we describe the clinical, biochemical and genetic characteristics of patients from 15 Iranian families with a clinical diagnosis of Bartter Syndrome.
\end{abstract}

Results: Age range of patients included in this study was 3 months to 6 years and all patients showed hypokalemic metabolic alkalosis. 3 patients additionally displayed hypercalciuria, with evidence of nephrocalcinosis in one case. Screening by Whole Exome Sequencing (WES) and long range PCR revealed that 12/17 patients (70\%) had a deletion of the entire CLCNKB gene that was previously identified as the most common cause of Bartter Syndrome in other populations. 4/17 individuals (approximately 25\% of cases) were found to suffer in fact from pseudo-Bartter syndrome resulting from congenital chloride diarrhea due to a novel homozygous mutation in the SLC26A3 gene, Pendred syndrome due to a known homozygous mutation in SLC26A4, Cystic Fibrosis (CF) due to a novel mutation in CFTR and apparent mineralocorticoid excess syndrome due to a novel homozygous loss of function mutation in HSD11B2 gene. 1 case (5\%) remained unsolved.

\footnotetext{
* Correspondence: dor_kordi@yahoo.com; dor_kordi@science.usb.ac.ir; miriam.schmidts@uniklinik-freiburg.de

2Departement of Biology, University of Sistan and Baluchestan, Zahedan, Iran ${ }^{1}$ Genome Research Division, Human Genetics department, Radboud University Medical Center, Geert Grooteplein Zuid 10, 6525KL Nijmegen, The Netherlands

Full list of author information is available at the end of the article
}

(c) The Author(s). 2019 Open Access This article is distributed under the terms of the Creative Commons Attribution 4.0 International License (http://creativecommons.org/licenses/by/4.0/), which permits unrestricted use, distribution, and reproduction in any medium, provided you give appropriate credit to the original author(s) and the source, provide a link to the Creative Commons license, and indicate if changes were made. The Creative Commons Public Domain Dedication waiver (http://creativecommons.org/publicdomain/zero/1.0/) applies to the data made available in this article, unless otherwise stated. 


\begin{abstract}
(Continued from previous page)
Conclusions: Our findings demonstrate deletion of CLCNKB is the most common cause of Bartter syndrome in Iranian patients and we show that age of onset of clinical symptoms as well as clinical features amongst those patients are variable. Further, using WES we were able to prove that nearly $1 / 4$ patients in fact suffered from Pseudo-Bartter Syndrome, reversing the initial clinical diagnosis with important impact on the subsequent treatment and clinical follow up pathway. Finally, we propose an algorithm for clinical differential diagnosis of Bartter Syndrome.
\end{abstract}

Keywords: Bartter syndrome, Whole exome sequencing, Pseudo-Bartter-syndrome

\section{Background}

Bartter Syndrome (BS), firstly reported by Bartter and his colleagues in 1962 , is a very rare autosomal recessive salt-losing tubulopathy characterized by hypokalemic metabolic alkalosis with normotensive hyperreninemia and hyperaldosteronism [1] occurring with an estimated incidence of $1.2 /$ million in the population [2]. Based on the loss of function mutations in the salt reabsorption transporters and channels in the thick ascending limb of the loop of Henle, genetically, five variants of this syndrome have been described: type I resulting from loss of function mutations in the Solute Carrier Family 12 Member 1 SLC12A1 gene encoding the apical furosemide-sensitive $\mathrm{Na}-\mathrm{K}-\mathrm{Cl}$ co-transporter (OMIM \#600839), type II caused by mutations in the potassium voltage-gated channel subfamily J member 1 (KCNJ1) gene encoding the apical renal outer medullary potassium channel (ROMK) (OMIM \# 600359), type III caused by mutations in the chloride voltage-gated channel $\mathrm{Kb}(C L C N K B)$ gene encoding the basolateral chloride channel Kb (OMIM \#602023), type IVa resulting from dysfunction of the Barttin CLCNK type accessory beta subunit (BSND) gene encoding Barttin, a subunit of chloride-channels $\mathrm{Ka}$ and $\mathrm{Kb}$ (OMIM \#606412) and finally, type IVb caused by co-mutation in the CLCNKA and CLCNKB genes (OMIM \#602024) [3-7]. Gitelman syndrome which shares several clinical characteristics with BS type III has been described later in history. In contrast to BS type III, Gitelman syndrome is caused by mutations in a single gene, SLC12A3 (OMIM \#263800), encoding the thiazide-sensitive sodium chloride co-transporter $(\mathrm{NCCT})$ in the distal convoluted tubule $[8$, 9]. More recently, 2 genes, namely calcium sensing receptor (CASR) (OMIM \#601198) and MAGE family member D2 (MAGED2) (OMIM \#300971, BS type V) have been identified which cause autosomal dominant and X-linked recessive forms of BS $[10,11]$.

Currently, from a phenotypical perspective, BS has been classified into three different forms according to the average age at onset of symptoms: antenatal BS, the most severe form of BS, marked by polyhydramnios, hypercalciuria, nephrocalcinosis, hypochloremia and failure to thrive in infancy; Second, classic BS which has a milder phenotype and is usually diagnosed during late adulthood. And third, Gitelman syndrome which compared to other variants is marked with hypocalciuria and hypomagnesaemia and is usually diagnosed during late childhood and adulthood. In most cases, BS IVa and IVb subtypes are accompanied by sensorineural deafness [12]. However, the BS subtype can often not be determined clinically with certainty due to similar presentation of different forms, rendering diagnostics and precise prognosis complex [13]. Despite the need for rigorous classification of BS phenotypes, currently few practical indicators exist.

Making the landscape of BS and BS-like clinical presentations appear even more complex, several renal and extra-renal disorders as congenital chloride diarrhea, Pendred syndrome, Cystic fibrosis as well as some acquired conditions associated (for example laxative abuse) may present clinically in a similar way as BS with regards to hypokalemic metabolic alkalosis. However, there is only a limited number of reports in the literature investigating misdiagnosis of BS where in fact other rare hypokalemic disorders (CF) were causative for the phenotype [14-22]. Nevertheless, such clinical misdiagnosis can result in serious health problems due to wrong treatment choices [23, 24]. However, novel high throughput sequencing technologies nowadays offer an additional diagnostic tool refining clinical diagnostics.

In the present study, we describe 17 patients from 15 Iranian families with a clinical diagnosis of BS. Implementing WES as additional diagnostic step combined with long-range PCR screening for $C L C N K B$, we identified the underlying genetic cause in $16 / 17$ cases. While we confirmed the clinical diagnosis of BS in 12 cases, our genetic analysis established a diagnosis different from BS in 4 cases. Additionally, we propose a cost-efficient clinical differential diagnostic algorithm.

\section{Results}

Clinical and genetics findings are summarized in Table 1. Laboratory results of all 17 patients showed severe hypokalemic alkalosis. Comparison of normal ranges of urinary calcium/creatinine ratio for age (hypercalciuria screening) indicated that case 3 showed hypocalciuria 


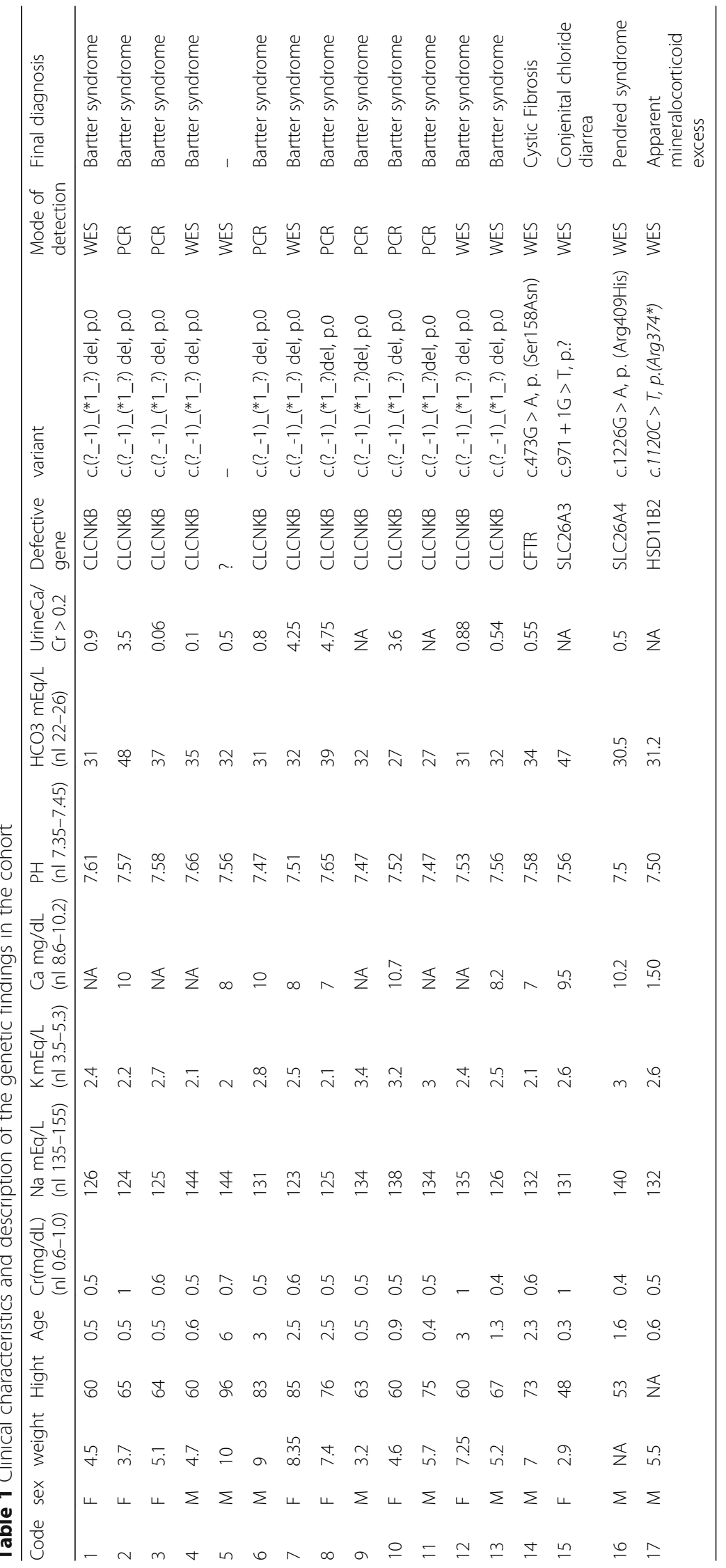


and case 2, 8, and 10 showed hypercalciuria (age dependent normal urinary creatinine/calcium ratios are shown in Table S2). 9 out of 17 cases clinically presented before age of 1 year, 2 out of 17 cases between 1 to 2 years old, 5 out of 17 cases between 2 to 3 years old and one case at the age of 6 years. Case 9 and case 15 did not survive due to severe hypokalemic metabolic alkalosis. In summary, we observed a spectrum of phenotypes ranging from BS type I to Gitelman syndrome in these families.

In order to investigate the underlying genetic causes, we proceeded with WES analysis in patient 13 as an index case. This revealed a deletion of the entire $C L C N K B$ gene (Fig. 1a). We therefore proceeded to check the remaining 16 patients by Sanger sequencing for this deletion. Due to high sequence similarity between $C L C N K A$ and $C L C N K B$, we used long range PCR generating a $C L C N K B$ specific gene product by using primer pairs with the forward primer in exon 9 and the reverse primer in exon 14. This confirmed a homozygous $C L C N K B$ in 11 out of the remaining 16 cases as well as the index case sent for WES initially. A total of 12 out of the 17 cases were found to carry the deletion (Fig. 1b).

The remaining 5 samples not found to carry the CLCNKB deletion were subsequently sent for WES. This revealed a novel homozygous CFTR missense mutation (NM_000492.3 (CFTR): c.473G > A, p. (Ser158Asn)) in case 14 . The mutated amino acid is highly conserved in 10 species down to zebrafish (Additional file 1: Figure S1) and the variant minor allele frequency is extremely low in control samples $(0.00007381$ in genomAD). Further, a different variant at the same position, CFTR c.473G $>$ C has been previously reported in a case of Cystic fibrosis [25]. The patient was a 27 months old boy with a clinical BS diagnosis living in the southeast of Iran where a hot and dry weather pattern is prevalent. His medical records revealed parental consanguinity and history of failure to thrive, delayed growth, muscle weakness, dehydration and polyuria. The diagnosis of BS had been established based on the electrolytes disturbance $\left(\mathrm{K}^{+}: 2.1 \mathrm{mEq} / \mathrm{L}\right)$, and blood gas analysis $\left(\mathrm{HCO}_{3}{ }^{-}: 34 \mathrm{mEq} / \mathrm{L}, \mathrm{PH}: 7.58\right)$. Treatment included $\mathrm{KCl}$ per $10 \mathrm{mEq} /$ day and he had not been investigated for $\mathrm{CF}$ due to lack of significant respiratory symptoms.

WES identified a novel homozygous canonical splice site mutation at the exon/intron boundary of exon 8 of the SLC26A3 gene (NM000111.2; c. $971+1 \mathrm{G}>\mathrm{T}$ ) for case 15. Recessive loss of function mutations in SLC26A3 have been previously reported to cause congenital chloride diarrhea [26]. This case was a 3 months old girl with a history of polyhydramnios, failure to thrive, dehydration and polyuria. At the time of birth, her body weight was $2.9 \mathrm{~kg}$, height was
$48 \mathrm{~cm}$, and head circumference was $33 \mathrm{~cm}$. After 3 months, she was hospitalized for delayed growth, muscle weakness and significant electrolyte imbalances. The diagnosis of BS had been established based on the electrolyte disturbance $\left(\mathrm{K}^{+}: 2,6 \mathrm{mEq} / \mathrm{L}\right)$, blood gas analysis $\left(\mathrm{HCO}_{3}^{-}: 47, \mathrm{PH}: 7,56\right)$ and she did not have a noted history of diarrhea. The treatment involved KCL ampoules $(10 \mathrm{mEq} /$ day $)$ under which she clinically improved.

For case 16, we detected a known homozygous missense mutation in the SLC26A4 gene by WES (NM_000441.1 (SLC26A4): c.1226G > A, p. (Arg409His), and rs111033305). This variant has been previously described as pathogenic in ClinVar (RCV000169222.1). The variant had previously been found in multiple Pendred patients, however not associated with hypokalemic metabolic alkalosis [27-29]. Our case was an 18 months old boy descending from consanguineous parents with a prenatal history of severe polyhydramnios, fetal distress and meconium in the amniotic fluid. Postnatally, failure to thrive with metabolic alkalosis, vomiting and elevated body temperature were noted. Besides, results of auditory brainstem response, the auditory steady-state response, oto-acoustic emission and tympanometry tests showed bilateral mild hearing loss. The diagnosis of BS had been established based on the electrolytes disturbance $\left(\mathrm{Cl}^{-}: 24 \mathrm{mEq} / \mathrm{L}, \mathrm{K}^{+}: 3\right.$ $\mathrm{mEq} / \mathrm{L})$, blood gas analysis $\left(\mathrm{HCO}_{3}{ }^{-}: 30.5, \mathrm{PH}: 7.50\right)$ and sensorial deafness. The stool exam showed no OVA, cyst and amoeba. The treatment involved $\mathrm{KCl}$ ampoules $(10 \mathrm{mEq} /$ day $)$ and Spironolactone $25 \mathrm{mg}$ every $8 \mathrm{~h}$.

Finally, WES revealed a novel homozygous loss of function mutation in HSD11B2 (NM_000196 (HSD11B2): c.1120C > T, p. $\left.\left(\operatorname{Arg} 374^{*}\right)\right)$, establishing the genetic diagnosis of apparent mineralocordicoid excess (AME). Our case was a 6 months old boy of consanguineous parents. At the time of examination his body weight was $5.50 \mathrm{~kg}$ with biography of failure to thrive, hypokalemic metabolic alkalosis, and also small stones in kidney sonography. Abdominal ultrasound did not reveal any abnormalities in the liver, pancreas, spleen and bladder. His blood pressure values were in normal range in the follow up exmaminations. The diagnosis of BS was suggested based on blood electrolytes disturbance $\left(\mathrm{K}^{+}: 2.6 \mathrm{mEq} / \mathrm{L}\right)$ and blood gas analysis $\left(\mathrm{HCO}_{3}{ }^{-}: 31.2\right.$, PH: 7.50).

Cases 5 remained unsolved.

Pedigrees of all 15 families are shown in Fig. 2, Normal ranges of urinary calcium/creatinine ratio in children is shown in Additional file 1: Figure S2, Sanger sequencing primers can be found in Additional file 1: Figure S3, sequencing results for family $12,13,14$ and 15 are shown in Additional file 1: Figure S4. 


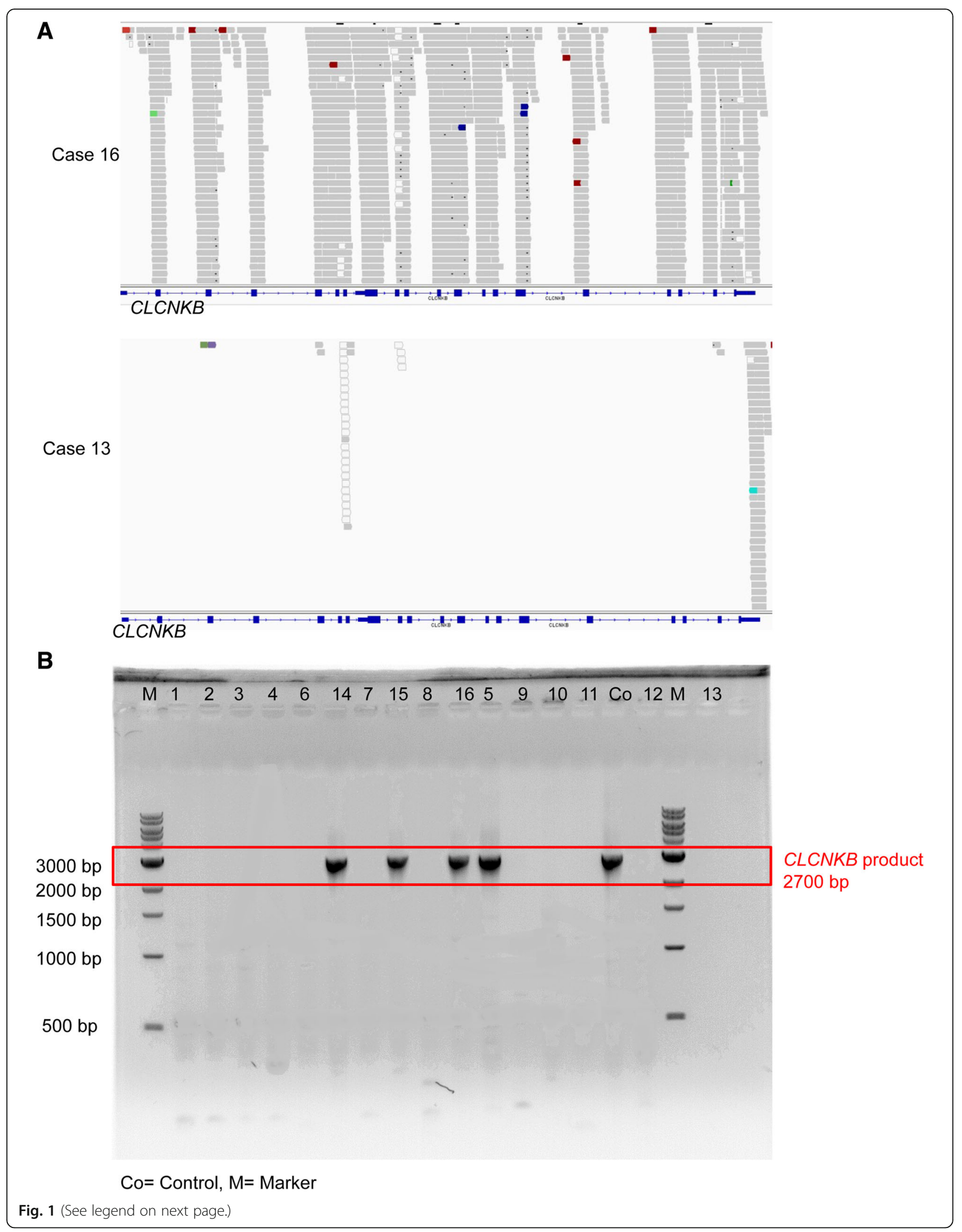


(See figure on previous page.)

Fig. 1 Visualization of CLCNKB deletion detected. (a) BAM files generated from WES data and displayed in IGV visualizing the deletion of the entire CLCNKB gene in case 13 (bottom) while reads are present in case 16 (top) who carries a homozygous mutation in SLC26A4. (b) Confirmation of CLCNKB deletion by long range PCR shown as absence of CLCNKB product in 12/16 patients on a $1 \%$ agarose gel containing ethidium bromide. $\mathrm{M}=$ marker, $\mathrm{Co}=$ control

\section{Discussion}

In this Iranian cohort, deletion of the entire $C L C N K B$ gene was identified as the most common allele causing BS. This is in line with previous findings in other populations where this deletion has also been found to represent the most common BS allele, especially in BS type III. In our cohort, phenotypes resulting from the deletion ranged from type I BS to Gitelman syndrome. All individuals in our cohort originated from the same region in the Southeast of Iran (Baloch ethnicity). It is possible families are remotely related and share a distant common ancestor. Interestingly, phenotypic presentations e.g. with regards to the age at onset of the first symptoms and presentations resembling different BS subtypes were highly variable amongst individuals harboring the identical $C L C N K B$ deletion. Intrafamilial phenotypic variability has been previously reported in a very large inbred Bedouin kindred in Northern Israel as well as a Spanish family [23, 30].

Putatively, the phenotypic differences observed could be caused by non-genetic factors e.g. environmental influences or result from the different genetic background between individuals (multigenic cause). Specifically, genotypic differences with regards to other (chloride) channels and transporters in the nephron resulting in different expression levels or function could also play a role (Fig. 3) [31, 32].

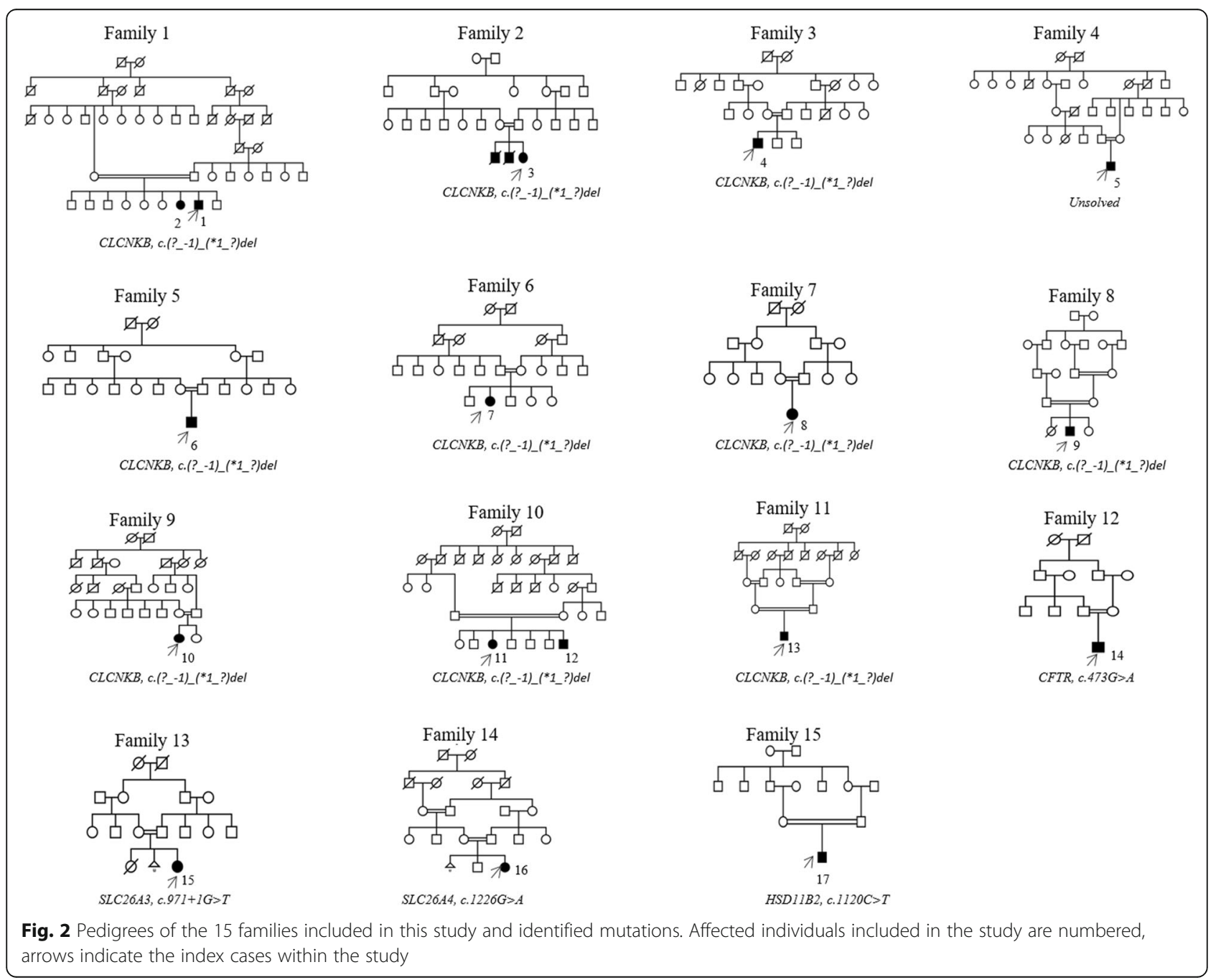




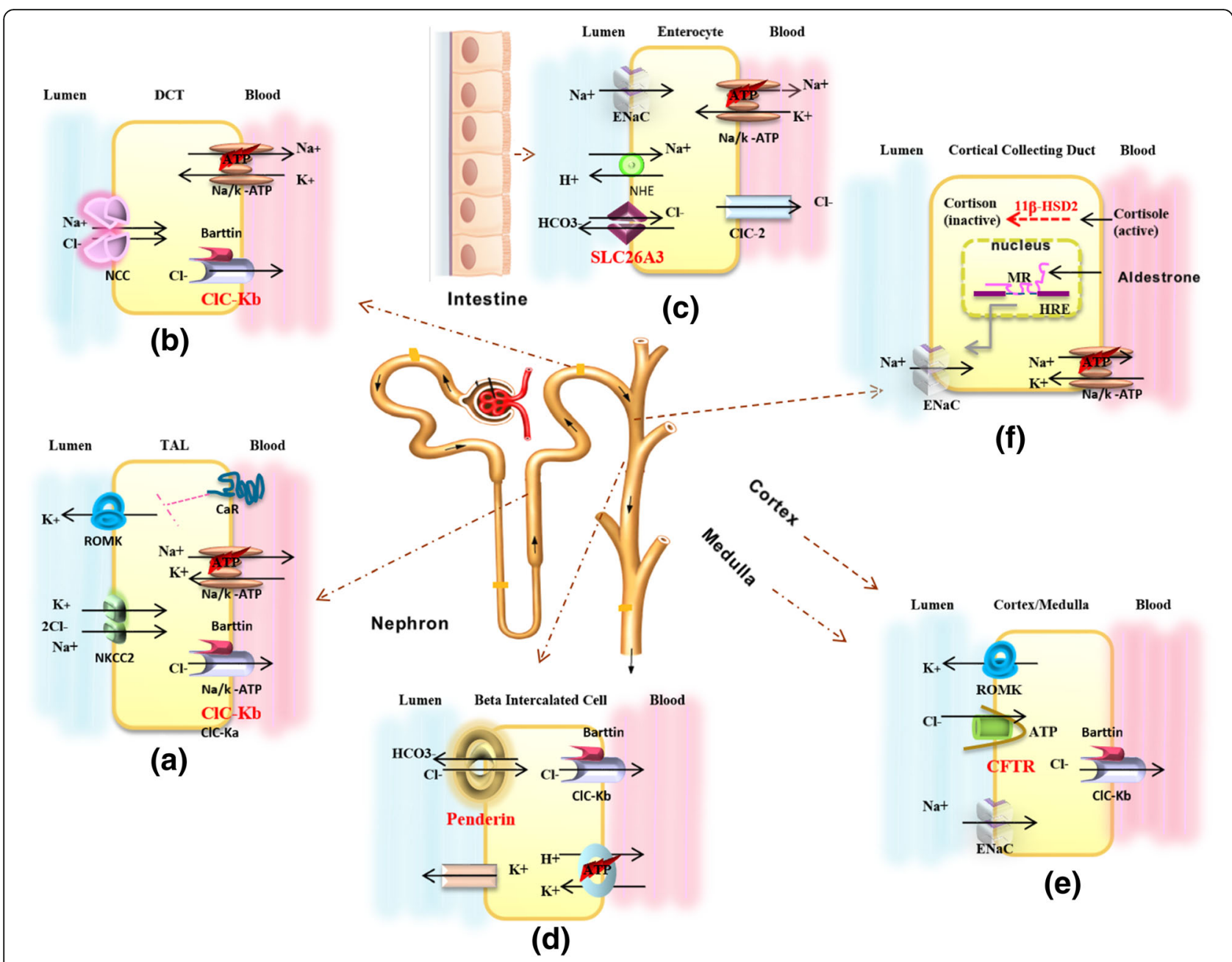

Fig. 3 Schematic of localizations and function of ion transporters defective in Bartter Syndrome and Pseudo-Bartter conditions. ClC-kb is mainly found in the thick ascending loop of Henle (TAL), (a) and distal tubules (DCT), (b) of kidneys, SLC26A3 in the intestine (c), Pendrin localizes mainly to renal beta-intercalated cells (d), CFTR is found in all nephron segments (e), and 11ß-HSD2 enzyme in cortical collecting duct (f). (a) Thick ascending loop of Henle: Luminal NKCC2 enables import of $\mathrm{Na}+, \mathrm{K}+$ and $\mathrm{Cl}$ - into the cells. K+ flows back to the lumen through ROMK1 channels; $\mathrm{Na}+$ and $\mathrm{Cl}$ - are reabsorbed to the blood stream through $\mathrm{Na}+/ \mathrm{K}+$ ATPase and CIC-kb channels. CASR inhibits the luminal ROMK channel which in turn results in decreased $\mathrm{NaCl}$ reabsorption and increased urinary $\mathrm{Cl}$-. (b) Distal Tubulus: $\mathrm{Cl}$ - transport occurs via the luminal, NCCT and exit to blood by $\mathrm{ClC}-\mathrm{kb}$. (c) In enterocytes, $\mathrm{Cl}$ - absorbed from the intestinal lumen via SLC26A3 and transported to the interstitium by $\mathrm{ClC}-2$. Na+ enters the cell via ENaC channels or $\mathrm{Na}+/ \mathrm{H}+$ exchangers and is transported to the interstitium by the $\mathrm{Na}+/ \mathrm{k}+\mathrm{ATPase}$. (d) Penderin participates in urinary bicarbonate excretion with tubular Cl- reabsorption. (e) CFTR functions as a Cl-channel and CFTR functions influences other ion channels such as ENaC and ROMK in the cortex and medulla. (f) mineralocorticoid aldosteron binds to Mineralcorticoid receptors (MR) which in turn binds to the hormone response elements (HRE) in the nucleus and stimulates increased resorption of $\mathrm{Na}$ + from the urine through transcription of genes involved in ENac and Na+/K+ ATP channels. Simultaneously glucocorticoid cortisol oxidized to inactive cortisone by $11 \beta$-HSD2 enzyme

To date, no larger studies have been published about the incidence of clinical misdiagnosis of BS where in fact other conditions are causative for the symptoms. Congenital chloride diarrhea results from loss of function mutations in the $S L C 26 \mathrm{~A} 3$ gene encoding an $\mathrm{Cl}^{-} / \mathrm{HCO}_{3}{ }^{-}$ exchanger in the intestine (Fig. 3c). Previous to our report, confounding congenital chloride diarrhea with BS has been reported in 2 additional cases without mentioning the underlying mutations $[14,15]$. Potentially, watery diarrhea could be confounded with urine and with increasing dehydration, the amount of diarrhea decreases, making a diagnosis of congenital chloride diarrhea even more difficult. Unlike in BS patients where high urinary $\mathrm{Cl}^{-}$concentration is found, low urinary and high fecal $\mathrm{Cl}^{-}$is detected in congenital chloride diarrhea [33]. Thus, both congenital chloride diarrhea and BS should be considered as differential diagnosis in patients with hypokalemic metabolic alkalosis. Untreated congenital chloride diarrhea can be lethal due to the acute and chronic dehydration and secondary impairment of renal function. Although by $\mathrm{KCl}$ or $\mathrm{NaCl}$ substitution electrolyte levels can be kept in balance, this does not influence the amount of diarrhea. 


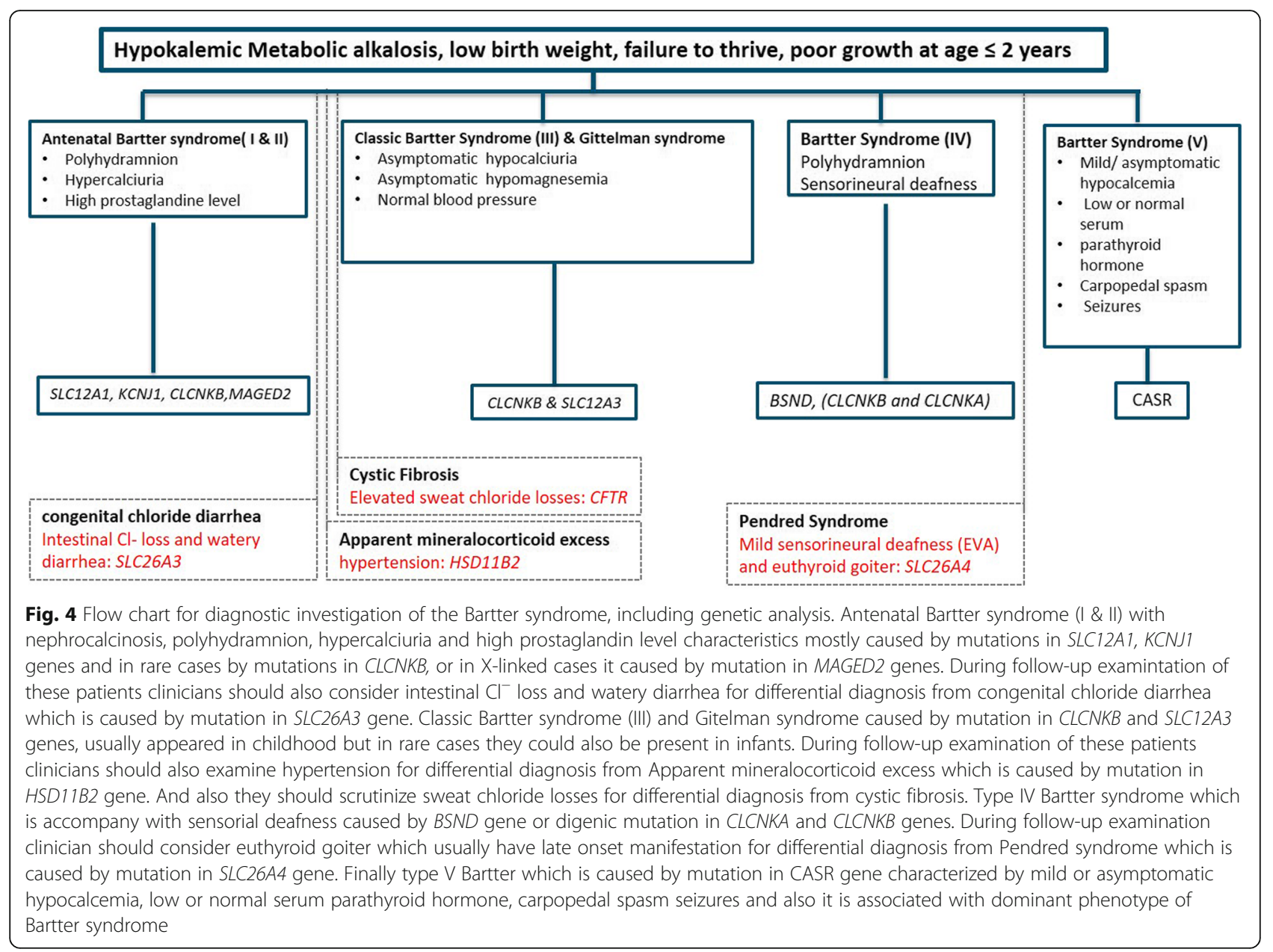

Second clinical misdiagnosis of BS in our cohort concerns a case which genetically turned out to represent Pendred syndrome. This syndrome is characterized by severe to mild hearing loss and euthyroid goiter [34]. Diagnosis of BS in this 18 months old was made base on hypokalemic metabolic alkalosis with sensorial deafness without any sign of euthyroid goiter. As goiter manifestation develops usually after the age of 10 years, Pendred syndrome can easily misdiagnosed in infants as our case [35]. Only a handful of patients with SLC26A4 mutations and metabolic alkalosis have been reported in the literature including a 46-year-old Caucasian female with sensorineural deafness and hypothyroidism $\left(\mathrm{Cl}^{-}: 86\right.$ $\left.\mathrm{mmol} / \mathrm{l}, \mathrm{K}^{+}: 1.4 \mathrm{mmol} / \mathrm{l}, \mathrm{HCO}_{3}{ }^{-}: 45 \mathrm{mmol} / \mathrm{l}\right)$, a child following thiazide therapy (potassium 1.7, chloride 70 , sodium 129, HCO3 43.8, base excess $+17.8 \mathrm{mmol} / \mathrm{l}$, $\mathrm{pH}$ 7.52 ), and another 46-year-old woman with sensorineural deafness, hypothyroidism, and profoundly low potassium levels $\left(\mathrm{K}^{+}: 1.4 \mathrm{mmol} / \mathrm{l}, \mathrm{HCO}_{3}{ }^{-}: 45 \mathrm{mmol} / \mathrm{l}\right)$ [16, $36,37]$. Pendred syndrome is caused by mutations in SLC26A4 encoding Pendrin which acts as $\mathrm{Cl}^{-} / \mathrm{HCO}_{3}{ }^{-}$ exchanger in the inner ear, thyroid and kidney [34].
Under basal conditions, Pendrin mediates acid- base balance through $\mathrm{HCO}_{3}{ }^{-}$excretion and $\mathrm{Cl}^{-}$reabsorption in the kidney in the $\beta$-intercalated cells of the cortical collecting duct in the kidney (Fig. 3d) [38]. Loss of function in the kidney is usually compensated by the other transporters; therefore, malfunction of Pendrin in the kidney does usually not result in detectable clinical symptoms. However, it is suggested that Pendrin might have an additional adaptive role in eliminating excess bicarbonate in alkalosis conditions [16]. Pendred syndrome therefore should be considered in infants or very young children with hypokalemic alkalosis and sensorial deafness alongside BS.

The third genetic revision of the initial clinical diagnosis in our cohort concerns a case with homozygous CFTR missense mutation manifesting as pseudo-BS. CFTR is a chloride channel expressed in many organs, including the kidneys. A number of publications have reported to date that in regions with hot climate, patients younger than 2 years with CF may present electrolyte disturbance without any other signs of respiratory and gastrointestinal abnormalities [39]. CFTR is expressed in all nephron segments 
where it is not only involved in $\mathrm{Cl}^{-}$transportation but also regulates other ion channels such as ENaC and ROMK through ATP transport (Fig. 3e) [40]. Several other pathogenic CFTR variants such as $3849+40 \mathrm{~A}>\mathrm{G}, 2.789+5$ G > A, F311 L, T3381, D110H, S13F, D110E, N1303K and $\triangle \mathrm{F} 508$ have been associated with hypokalemic metabolic alkalosis [41]. To our knowledge, the c.473G > A mutation has not been reported yet to manifest only with pseudo-BS in the absence of respiratory and gastrointestinal symptoms. However, in hot weather, like in southern Iran, high sweat production rate lead to hypokalemia in sweat and urine through massive $\mathrm{NaCl}$ loss and secondary hyperaldosteronism Hypokalemic metabolic alkalosis is observed in both BS and CF patients, but urinary chloride loss in BS syndrome is higher than in CF patients.

In the fourth case with a clinical misdiagnosis of BS, we identified ahomozygous $H S D 11 B 2$ mutation, reversing the diagnosis to AME. So far, fewer than 100 AME cases have been reported in the literature. The clinical hallmark of this disorder is hypokalemic metabolic alkalosis with severe childhood- or juvenile-onset hyporeninemic hypertension [41]. Under normal conditions, the mineralocorticoid aldosteron binds to mineralcorticoid receptors (MR) which in turn bind to hormone response elements (HRE) in the nucleus, resulting in transcription of ENac and $\mathrm{Na}^{+} / \mathrm{K}^{+}$ATP channels in renal collecting duct cells. Simultaneously, the glucocorticoid cortisol which has similar MR affinity as aldosterone is oxidized to the inactive form cortisone by the $11 \beta-H S D 2$ enzyme (Fig. 3f), inhibiting binding to MR. Under conditions of impaired 11 $\beta$-HSD2 function, cortisol which is present in 1000-2000 times higher concentrations compared to aldosterone, binds to MR, causing enhanced $\mathrm{Na}^{+}{ }^{-}$reabsorption which in turn leads to the expansion of intravascular fluid, causing hypertension [42]. Diagnosis of BS in the 6 month old patient described here was made based on hypokalemic metabolic alkalosis with low birth weight, failure to thrive and poor growth. Hypertension however only occurs later during the course of AME, thus does not help to differentiate AME from BS in toddlers. [41, 43]. In addition, because the incidence of hypertension is low in children less than three years of age, routine blood pressure monitoring is not recommended unless the patient is at risk for hypertension, facilitating misdiagnosis of AME.

In summary, clinical misdiagnosis of BS in our cohort was nearly $25 \%$. This suggests a generally high chance for BS misdiagnosis, especially in developing countries where elaborated biochemical analysis is not available due to considerable phenotypic overlap between different rare hypokalemic disorders. Additionally, current clinical classification of antenatal BS, classic BS and Gitelman syndrome is complicated and not always specific. We found $C L C N K B$ deletions to cause a broad phenotypic spectrum. A clinical diagnostic algorithm is proposed in Fig. 4 for patients with hypokalemic metabolic alkalosis, low birth weight and failure to thrive within the first 2 years of life. Common biochemical markers related to different types of Bartter syndrome, also watery diarrhea, hypertension, sweat chloride losses, enlarged vestibular aqueducts (EVA) visible in CT-scans and euthyroid goiter should be actively looked out for.

However, NGS approaches now allow time and cost efficient parallel analysis of several (or all) coding genes, offering additional diagnostic possibilities, independent of correct clinical phenotype classification. In this study, WES costs per sample were 250 USD including bioinformatics (Novogene, Hongkong), not including variant filtering performed in-house using the variant files provided by the company. Wider implementation of WES will hopefully result in more precise diagnosis and targeted treatment approaches as well as new genetic counseling opportunities, especially in countries with limited biochemical testing facilities.

\section{Conclusions}

In summary, our data suggests that BS patients negative for the common $C L C N K B$ deletion profit from WES and that Pseudo-BS e.g. due to $\mathrm{CF}$, congenital chloride diarrhea, Pendred Syndrome and AME should be considered as differential diagnosis in infants and young children with hypokalemic metabolic alkalosis.

\section{Methods}

\section{Human research subjects}

Consent forms were obtained from all the participants of this study. Ethical approval committee of Mashhad University of medical sciences approved this study (IR.MUMS.REC.1395.534). 17 patients from 15 families referred to our clinics from 2016 to 2018 with diagnosis of BS were included. The basic information and laboratory results of the patients have been summarized in Table 1 and pedigrees have been shown in Fig. 1 .

\section{DNA extraction}

Genomic DNA was extracted from whole blood, using standard salting out method. The concentration of DNA was measured by Qubit 2.0 (life technologies, Carlsbad, CA, USA).

\section{WES}

2 microgram DNA from 6 patients out of 17 patients [5, 13-17], and was used for WES, using Illumina HiSeq 2500, Q30 $\geq 80 \%$ (Novogene, Hongkong). Exome capture was performed with Agilent SureSelect Human All Exon V6 Kit, sequencing depth was 50x using paired-end sequencing on a HiSeq 2500 Genome Analyzer (Illumina), resulting in sequences 
of 150 bases from each end of the fragments. UCSC hg19 was used as a reference genome. VarScan version 2.2.5 and MuTec and GATK Somatic Indel Detector were used to detect SNV and InDels, respectively. Data was filtered for $\mathrm{MAF}<1 \%$ in public control databases such as dbSNP, ExAc and gnomad (gnomAD, http://gnomad.broadinstitute.org). Additionally, variants occurring with MAF $>0.01$ in the Iranome (http://www.iranome.ir) were also excluded. The remaining variants were filtered for known disease causing genes first and we prioritized homozygous variants due to the autosomal recessive inheritance pattern of disease and consanguinity. For families without plausible variants left after filtering, BAM files were visually inspected for homozygous CNVs in known disease causing genes related to a hypokalemic metabolic phenotype.

\section{PCR and sanger sequencing}

Conventional PCR was done by Taq polymerase (Roche, Mannheim, Germany) based on manufacture's instruction to extend the mutated region with specific primer. Also, long range PCR (200 bp- $4 \mathrm{~kb}$ ) was done by AccuPrime $^{\text {tr }}$ Taq DNA polymerase system to confirm deletion in $C L C N K B$ gene with specific primer according to the following condition, $2.5 \mu \mathrm{l}$ of $10 \mathrm{x}$ AccuPrime $^{\mathrm{Tu}}$ PCR Buffer II, $0.5 \mu \mathrm{l}$ of Primer Mix (10 $\mu \mathrm{M}$ each), 1-200 ng of template DNA, $0.5 \mu \mathrm{l}$ of AccuPrime ${ }^{\mathrm{Tw}}$ Taq DNA polymerase in total of 25 autoclaved distilled water (initial denaturation at $94^{\circ} \mathrm{C}$ for $2 \mathrm{~min}$; followed by 40 cycles of denaturing at $94{ }^{\circ} \mathrm{C}$ for $30 \mathrm{~s}$, annealing at $65{ }^{\circ} \mathrm{C}$ for 30 s, and extension at $68{ }^{\circ} \mathrm{C}$ for $3 \mathrm{~min}$ and final extension at $68{ }^{\circ} \mathrm{C}$ for $5 \mathrm{~min}$ ). Primer sequences are in Table S1. Before Sanger sequencing, PCR products were cleaned by ExoSAP-IT ${ }^{\bullet}$ (USB, Cleveland, Ohio, USA). The cleanup PCR products were bidirectional sequenced using 3730XL DNA analyzer (ABI, Foster City).

\section{Web resources}

Homozygosity-Mapper, http://www.homozygositymapper.org/.

Exome Aggregation Consortium (ExAC), http://exac.broadinstitute.org/.

Genome Aggregation Database (gnomAD), http://gnomad.broadinstitute.org/.

dbSNP, http://www.ncbi.nlm.nih.gov/SNP/.

1000 Genomes Project human polymorphism database, http://www.1000genomes.org/.

National Heart, Lung and Blood Institute-Exome Sequencing Project, http://evs.gs.washington.edu/EVS/.

Online Mendelian Inheritance in Man, http://www. omim.org/.

Integrated genome viewer (IGV), http://software.broad institute.org/software/igv/.

\section{Additional file}

Additional file 1: Figure S1. Aminoacid conservation of CFTR p.Ser158Asn. Figure S2. Normal ranges of urinary calcium/creatinine ratio in children. Figure S3. Sanger sequencing primers used. Figure S4.

Sanger traces of identified mutations in non-Bartter-Syndrome genes. (DOCX 648 kb)

\section{Abbreviations}

AME: Apparent mineralocorticoid excess; BS: Bartter Syndrome; BSND: Barttin CLCNK type accessory beta subunit; CASR: Calcium sensing receptor; CFTR: Cystic fibrosis transmembrane conductance regulator;

CLCNKB: Chloride voltage-gated channel Kb; DCT: Distal convoluted tubule; HRE: Hormone Response Elements; KCNJ1: potassium voltage-gated channel subfamily J member 1; MAGED2: MAGE family member D2;

MR: Mineralcorticoid receptors; NCC: renal thiazide-sensitive $\mathrm{NaCl}$ cotransporter; NKCC2: Na-K-Cl cotransporter; PTC: Proximal tubular cells; ROMK: Renal outer medullary potassium channel; SLC12A1: Solute Carrier Family 12 Member 1; SLC26A3: Solute Carrier Family 26 Member 3; SLC26A4: Solute Carrier Family 26 Member 4; TAL: Thick ascending limb; WES: Whole exome sequencing

\section{Acknowledgments}

We would like to thank all participating families and clinicians, especially the Department of Biology, University of Sistan and Baluchestan, Iran, Ali Ebne Abitaleb Hospital in Zahedan, Iran and Dr. Sheikh Hospital in Mashhad, Iran for supporting this project.

\section{Consent to participate}

Consent forms were completed by all the participants of this study.

\section{Funding}

MS acknowledges funding from Radboudumc and RIMLS Nijmegen (Hypatia tenure track fellowship), the "Deutsche Forschungsgemeinschaft" (DFG CRC1140 KIDGEM) and the European Research Council (ERC StG TREAT Cilia, grant No 716344).

\section{Availability of data and materials}

Supporting information is supplied in the supplementary file. WES datasets have not been deposited due to rare variants described in this manuscript which could lead to identification of individuals.

\section{Authors' contributions}

MN performed sample preparation and Sanger sequencing and MN and MS performed WES data analysis. ZB helped with logistics. IS was involved in clinical interpretation. FB, SB, EGK and AA were involved in patient recruitment and clinical data collection. DMKT and MS conceived and supervised the study. MN and MS wrote the manuscript and all authors approved the final manuscript version.

Ethics approval and consent to participate

Ethical approval committee of Mashhad University of medical sciences approved this study (IR.MUMS.REC.1395.534).

\section{Consent for publication}

Patients have consented for all clinical data to be published.

\section{Competing interests}

The authors declare that they have no competing interests.

\section{Publisher's Note}

Springer Nature remains neutral with regard to jurisdictional claims in published maps and institutional affiliations.

\section{Author details}

${ }^{1}$ Genome Research Division, Human Genetics department, Radboud University Medical Center, Geert Grooteplein Zuid 10, 6525KL Nijmegen, The Netherlands. ${ }^{2}$ Departement of Biology, University of Sistan and Baluchestan, Zahedan, Iran. ${ }^{3}$ Genetics Research Center, University of Social Welfare and 
Rehabilitation Sciences, Tehran, Iran. ${ }^{4}$ Children and Adolescents Health Research Center, resistant tuberculosis institute, Zahedan University of Medical Sciences, Zahedan, Iran. ${ }^{5}$ Razavi Cancer Research, Razavi Hospital, Imam Reza International University, Mashhad, Iran. ${ }^{6}$ Next Generation Genetic Polyclinic, Mashhad, Iran. 'Department of Pediatrics, Faculty of Medicine, Mashhad University of Medical Sciences, Mashhad, Iran. ${ }^{8}$ Center for Pediatrics and Adolescent Medicine, Freiburg University Hospital, Freiburg University Faculty of Medicine, Mathildenstrasse 1, 79112 Freiburg, Germany. ${ }^{9}$ Center for Pediatrics and Adolescent Medicine, University Hospital Freiburg, 79112 Freiburg, Germany.

\section{Received: 23 June 2018 Accepted: 14 December 2018} Published online: 13 February 2019

\section{References}

1. Bartter FC, Pronove P, Gill JR Jr, MacCardle RC. Hyperplasia of the juxtaglomerular complex with hyperaldosteronism and hypokalemic alkalosis: a new syndrome. Am J Med. 1962;33(6):811-28.

2. Bokhari SRA, Mansur A: Bartter Syndrome.In StatPearls. Treasure Island (FL): StatPearls Publishing LLC; 2018.

3. Simon DB, Karet FE, Hamdan JM, Di Pietro A, Sanjad SA, Lifton RP. Bartter's syndrome, hypokalaemic alkalosis with hypercalciuria, is caused by mutations in the Na-K-2Cl cotransporter NKCC2. Nat Genet. 1996;13(2):183.

4. Simon DB, Karet FE, Rodriguez-Soriano J, Hamdan JH, DiPietro A, Trachtman $\mathrm{H}$, et al. Genetic heterogeneity of Barter's syndrome revealed by mutations in the K+ channel, ROMK. Nat Genet. 1996:14(2):152.

5. Simon DB, Bindra RS, Mansfield TA, Nelson-Williams C, Mendonca E, Stone R, et al. Mutations in the chloride channel gene, CLCNKB, cause Bartter's syndrome type III. Nat Genet. 1997;17(2):171.

6. Birkenhäger R, Otto E, Schürmann MJ, Vollmer M, Ruf E-M, Maier-Lutz I, et al, Mutation of BSND causes Bartter syndrome with sensorineural deafness and kidney failure. Nat Genet. 2001;29(3):310.

7. Nozu K, Inagaki T, Fu XJ, Nozu Y, Kaito H, Kanda K, et al. Molecular analysis of digenic inheritance in Bartter syndrome with sensorineural deafness. J Med Genet. 2008;45(3):182-6.

8. Gitelman HJ, Graham JB. Welt LG. A new familial disorder characterized by hypokalemia and hypomagnesemia. Trans Assoc Am Phys. 1966:79:221-35.

9. Kurtz I, Cohen JJ, Harrington JT, Madias NE, Zusman CJ. Molecular pathogenesis of Bartter's and Gitelman's syndromes. Kidney Int. 1998;54(4):1396-410.

10. Watanabe S, Fukumoto S, Chang H, Takeuchi Y, Hasegawa Y, Okazaki R, et al. Association between activating mutations of calcium-sensing receptor and Bartter's syndrome. Lancet. 2002;360(9334):692-4.

11. Laghmani K, Beck BB, Yang S-S, Seaayfan E, Wenzel A, Reusch B, et al. Polyhydramnios, transient antenatal Bartter's syndrome, and MAGED2 mutations. N Engl J Med. 2016;374(19):1853-63.

12. Konrad M, Vollmer M, Lemmink HH, Van den Heuvel LP, Jeck N, VargasPoussou R, et al. Mutations in the chloride channel gene CLCNKB as a cause of classic Bartter syndrome. J Am Soc Nephrol. 2000;11(8):1449-59.

13. Al Shibli A, Narchi H. Bartter and Gitelman syndromes: Spectrum of clinical manifestations caused by different mutations. World J Methodol. 2015:5(2):55-61.

14. Eğrıtaş $\mathrm{O}$, Dalgic B, Wedenoja S. Congenital chloride diarrhea misdiagnosed as Bartter syndrome. The Turkish journal of gastroenterology: the official journal of Turkish society of. Gastroenterology. 2011;22(3):321-3.

15. Saneian H, Bahraminia E. Congenital chloride diarrhea misdiagnosed as pseudo-Bartter syndrome. Journal of research in medical sciences: the official journal of Isfahan University of Medical. Sciences. 2013;18(9):822.

16. Kandasamy N, Fugazzola L, Evans M, Chatterjee K, Karet F. Lifethreatening metabolic alkalosis in Pendred syndrome. Eur J Endocrinol. 2011;165(1):167-70.

17. Bates CM, Baum M, Quigley R. Cystic fibrosis presenting with hypokalemia and metabolic alkalosis in a previously healthy adolescent. J Am Soc Nephrol. 1997:8(2):352-5.

18. Dahabreh MM, Najada AS. Pseudo-bartter syndrome, pattern and correlation with other cystic fibrosis features. Saudi J Kidney Dis Transpl. 2013;24(2):292-6.

19. Galaviz-Ballesteros MJ, Acosta-Rodriguez-Bueno CP, Consuelo-Sanchez A Franco-Alvarez I, Olalla-Mora Ol, Vazquez-Frias R. Pseudo-Bartter syndrome as manifestation of cystic fibrosis with DF508 mutation. Bol Med Hosp Infant Mex. 2016;73(5):331-4.
20. Tinsa F, Hadj Fredj S, Bel Hadj I, Khalsi F, Abdelhak S, Boussetta K, et al. Pseudo-Bartter syndrome as the sole manifestation of cystic fibrosis in a child with 711+G>T/IVS8-5T mutation: a new face of an old disease. Ann Biol Clin. 2017;75(4):466-73.

21. Qiu L, Yang F, He Y, Yuan H, Zhou J. Clinical characterization and diagnosis of cystic fibrosis through exome sequencing in Chinese infants with Barttersyndrome-like hypokalemia alkalosis. Front Med. 2018:1-9.

22. Baumner S, Weber LT. Nephropathic Cystinosis: symptoms, treatment, and perspectives of a systemic disease. Front Pediatr. 2018;6:58.

23. Garcia Castano A, Perez de Nanclares G, Madariaga L, Aguirre M, Madrid A, Chocron S, et al. Poor phenotype-genotype association in a large series of patients with type III Bartter syndrome. PLoS One. 2017;12(3):e0173581.

24. Bettinelli A, Vezzoli G, Colussi G, Bianchetti MG, Sereni F, Casari G. Genotypephenotype correlations in normotensive patients with primary renal tubular hypokalemic metabolic alkalosis. J Nephrol. 1998;11(2):61-9.

25. McGinniss MJ, Chen C, Redman JB, Buller A, Quan F, Peng M, et al. Extensive sequencing of the CFTR gene: lessons learned from the first 157 patient samples. Hum Genet. 2005;118(3-4):331-8.

26. Makela S, Kere J, Holmberg C, Hoglund P. SLC26A3 mutations in congenital chloride diarrhea. Hum Mutat. 2002;20(6):425-38

27. Rendtorff ND, Schrijver I, Lodahl M, Rodriguez-Paris J, Johnsen T, Hansen EC, et al. SLC26A4 mutation frequency and spectrum in 109 Danish Pendred syndrome/DFNB4 probands and a report of nine novel mutations. Clin Genet. 2013;84(4):388-91.

28. Guo YF, Liu XW, Guan J, Han MK, Wang DY, Zhao YL, et al. GJB2, SLC26A4 and mitochondrial DNA A1555G mutations in prelingual deafness in northern Chinese subjects. Acta Otolaryngol. 2008;128(3):297-303.

29. Blons H, Feldmann D, Duval V, Messaz O, Denoyelle F, Loundon N, et al. Screening of SLC26A4 (PDS) gene in Pendred's syndrome: a large spectrum of mutations in France and phenotypic heterogeneity. Clin Genet. 2004;66(4):333-40

30. Zelikovic I, Szargel R, Hawash A, Labay V, Hatib I, Cohen N, et al. A nove mutation in the chloride channel gene, CLCNKB, as a cause of Gitelman and Bartter syndromes. Kidney Int. 2003;63(1):24-32.

31. Zelikovic I. Hypokalaemic salt-losing tubulopathies: an evolving story. Nephrol Dial Transplant. 2003;18(9):1696-700.

32. Gorgojo JJ, Donnay S, Jeck N, Konrad M. A Spanish founder mutation in the chloride channel gene, CLCNKB, as a cause of atypical Bartter syndrome in adult age. Horm Res. 2006;65(2):62-8.

33. Hoglund P, Sormaala M, Haila S, Socha J, Rajaram U, Scheurlen W, et al. Identification of seven novel mutations including the first two genomic rearrangements in SLC26A3 mutated in congenital chloride diarrhea. Hum Mutat. 2001;18(3):233-42.

34. Wemeau JL, Kopp P. Pendred syndrome. Best Pract Res Clin Endocrinol Metab. 2017:31(2):213-24

35. Reardon W, Coffey R, Chowdhury T, Grossman A, Jan H, Britton K, et al. Prevalence, age of onset, and natural history of thyroid disease in Pendred syndrome. J Med Genet. 1999;36(8):595-8.

36. Pela I, Bigozzi M, Bianchi B. Profound hypokalemia and hypochloremic metabolic alkalosis during thiazide therapy in a child with Pendred syndrome. Clin Nephrol. 2008;69(6):450-3.

37. Sanei-Moghaddam A, Wilson T, Kumar S, Gray R. An unfortunate case of Pendred syndrome. J Laryngol Otol. 2011;125(9):965-7.

38. Soleimani M. The multiple roles of pendrin in the kidney. Nephrol Dial Transplant. 2015:30(8):1257-66

39. Vilotijevic-Dautovic G, Stojanovic V. Pseudo-Bartter's syndrome in patients with cystic fibrosis: a case series and review of the literature. Srp Arh Celok Lek. 2015;143(11-12):748-51.

40. Souza-Menezes J, Morales MM. CFTR structure and function: is there a role in the kidney? Biophys Rev. 2009;1(1):3-12.

41. Yau M, Haider S, Khattab A, Ling C, Mathew M, Zaidi S, et al. Clinical, genetic, and structural basis of apparent mineralocorticoid excess due to 11 beta-hydroxysteroid dehydrogenase type 2 deficiency. Proc Natl Acad Sci U S A. 2017;114(52):E11248-e56.

42. Kosicka K, Cymerys M, Majchrzak-Celinska A, Chuchracki M, Glowka FK. 11 beta-Hydroxysteroid dehydrogenase type 2 in hypertension: comparison of phenotype and genotype analysis. J Hum Hypertens. 2013;27(8):510-5.

43. Morineau G, Sulmont V, Salomon R, Fiquet-Kempf B, Jeunemaitre X, Nicod J, et al. Apparent mineralocorticoid excess: report of six new cases and extensive personal experience. J Am Soc Nephrol. 2006;17(11):3176-84. 